\title{
Review: behavioural interventions show the most promise for chronic fatigue syndrome
}

\author{
Whiting P, Bagnall AM, Sowden AJ, et al. Interventions for the treatment and management of chronic fatigue syndrome. A \\ systematic review.JAMA 2001 Sep 19;286:1360-8.
}

\section{QUESTION: In patients with chronic fatigue syndrome (CFS), what is the effectiveness of evaluated interventions?}

\section{Data sources}

Published and unpublished studies in any language were identified by searching 19 databases, including Medline, EMBASE/Excerpta Medica, PsycLIT, ERIC, Current Contents, and the Cochrane Library (to 2000). The internet was searched using a meta-search engine; references of retrieved articles were scanned; and individuals and organisations were contacted through a website dedicated to the review and through members of 2 advisory panels.

\section{Study selection}

Studies were selected if they were randomised controlled trials (RCTs) or controlled clinical trials of any intervention used in the treatment or management of CFS in adults or children. Studies in which diagnoses were based on another syndrome with criteria similar to CFS, such as those of myalgic encephalomyelitis, the chronic fatigue immune deficiency syndrome, or chronic Epstein-Barr virus infection, were included, but studies of fibromyalgia were not.

\section{Data extraction}

Data were extracted on study validity (allocation concealment [RCTs], control group, and adjustment for confounders [controlled studies]), baseline comparability of groups, blinding, follow up, drop outs, objectivity of outcome assessment, analysis, sample size, and cointerventions; intervention; diagnostic criteria; duration of follow up; and outcomes (psychological, physical, quality of life and health status, physiological, and resource use).

\section{Main results}

44 studies were included (32 studies enrolled adults, 1 enrolled children, and 2 enrolled adults and children; 9 studies did not give age information) $(\mathrm{n}=2801$; age range
11 to $87 \mathrm{y}, 71 \%$ women) with 31 different interventions; 36 studies were RCTs. The studies were grouped by type of intervention (behavioural, immunological, pharmacological, supplements, complementary or alternative, and other interventions). 18 trials $(41 \%)$ showed an overall beneficial effect of the intervention $(\geqslant 1$ clinical outcome improved). The results from the RCTs are in the table. Cognitive behavioural therapy and graded exercise treatment showed beneficial effects. Overall evidence from the other interventions was inconclusive.

\section{Conclusions}

In patients with the chronic fatigue syndrome, 31 different interventions show mixed results for effectiveness. Cognitive behavioural therapy and graded exercise treatment show the most promise.

Interventions from RCTs for the chronic fatigue syndrome*

\begin{tabular}{lcll} 
Interventions & $\begin{array}{c}\text { Number } \\
\text { of RCTs }\end{array}$ & $\begin{array}{l}\text { Number of } \\
\text { patients }\end{array}$ & $\begin{array}{l}\text { Overall effect } \\
\text { (improvement } v \\
\text { no difference) }\end{array}$ \\
Behavioural & 8 & 883 & $6 v 2$ \\
\hline Graded exercise & 3 & 350 & $3 v 0$ \\
\hline CBT & 5 & 533 & $3 v 2$ \\
\hline Immunological & 9 & 440 & $4 v 5$ \\
\hline Pharmacological & 12 & 896 & $2 v 10$ \\
\hline $\begin{array}{l}\text { Supplements } \\
\text { Complementary or } \\
\text { alternative }\end{array}$ & 5 & 174 & $2 v 3$ \\
\hline
\end{tabular}

${ }^{*} \mathrm{CBT}=$ cognitive behavioural therapy; $\mathrm{RCTs}=$ randomised controlled trials Intervention duration ranged from 2 weeks to 1 year (mean 16 wks); follow up ranged from 2 weeks to 5 years.

\section{COMMENTARY}

The well done review by Whiting et al does not help physicians much. In the included studies, the treatment of CFS was done by mental health and other specialists. Thus, as physicians, we must consider the data in regards to whether to refer. Qualified support exists for graded exercise treatment and cognitive behavioural therapy, but it is unknown whether the treatment effect lasts longer than a few months, and some studies reported high drop out rates.

The absence of a standard definition of CFS jeopardises interpretation. Many researchers have found so much overlap with other syndromes, such as the irritable bowel syndrome and fibromyalgia, that they posit that any definition of CFS is meaningless. ${ }^{2}$ They suggest we view syndromes like CFS as artifacts of specialisation that obscure the true problem of medically unexplained symptoms, which is the appropriate focus for our research and therapeutic efforts. ${ }^{12}$

I was struck by the erratically positive results across many unrelated studies. I suggest that the provider-patient relationship is the common feature, although it was not reported by the author and I have never seen it reported with controlled interventions in these populations; however, variations in the relationship may account for the variably positive results with so many unrelated treatments. The provider-patient relationship, in any event, is an appropriate focus for physicians in managing patients with CFS and can be useful in arranging referral to mental health specialists. ${ }^{3}$

Robert C Smith, MD Michigan State University East Lansing, Michigan, USA

1 Wessely S, Nimnuan C, Sharpe M. Functional somatic syndromes: one or many? Lancet 1999;354:936-9.

2 Aaron LA, Burke MM, Buchwald D. Overlapping conditions among patients with chronic fatigue syndrome, fibromyalgia, and temporomandibular disorder. Arch Intern Med 2000;160:221-7.

3 Smith RC. Somatization disorder: defining its role in clinical medicine.J Gen Intern Med 1991;6:168-75. 